\title{
Erratum
}

\section{Asteroid (216) Kleopatra}

\section{Tests of the radar-derived shape model}

\author{
D. Hestroffer ${ }^{1}$, J. Berthier ${ }^{1}$, P. Descamps ${ }^{1}$, P. Tanga ${ }^{2, \star}$, A. Cellino ${ }^{2}$, M. Lattanzi ${ }^{2}$, \\ M. Di Martino ${ }^{2}$, and V. Zappalà ${ }^{2}$ \\ 1 IMCCE, UMR CNRS 8028, Observatoire de Paris, 77 Av. Denfert Rochereau, 75014 Paris, France \\ 2 Osservatorio Astronomico di Torino (OATo), Strada Osservatorio 20, 10025 Pino Torinese (TO), Italy
}

Figure 1 has been misprinted so we print it again.

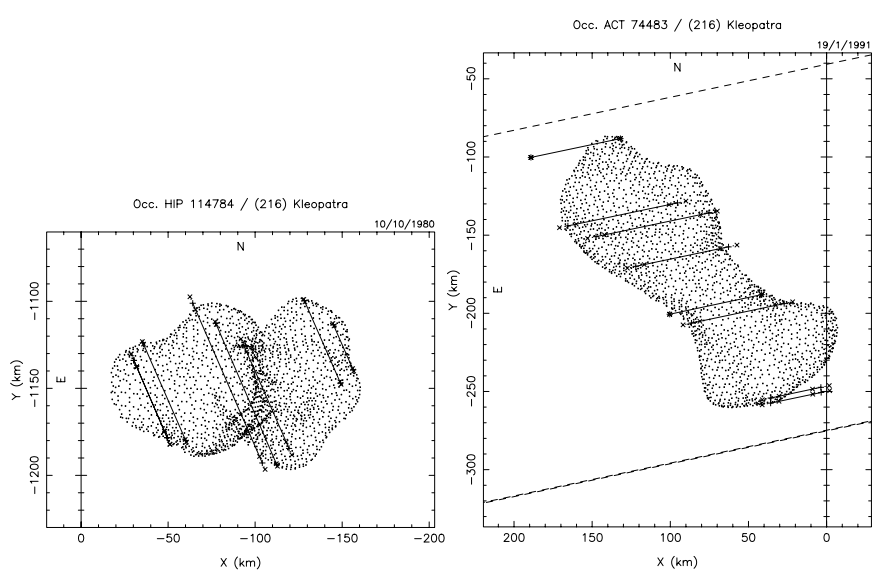

Fig. 1. Comparison between the observed occultation data and the radar model. Left panel: occultation of Oct. 10, 1980 at $7.00 \mathrm{~h}$ UTC. Right panel: occultation of Jan. 1, 1991 at $5.28 \mathrm{~h}$ UTC. The dashed lines correspond to negative observations. Error bars are given by crosses and are negligible for the photoelectric observations.

Send offprint requests to: D. Hestroffer, e-mail: hestro@bdl . fr

* Associate researcher at the IMCCE. Present address: Laboratoire Cassini, UMR 6529, Observatoire de la Côte d'Azur, BP 4229, 06304 Nice Cedex 4, France. 